\title{
SPEXOR: Spinal Exoskeletal Robot for Low Back Pain Prevention and Vocational Reintegration
}

\author{
Jan Babič, Katja Mombaur, Dirk Lefeber, Jaap van Dieën, \\ Bernhard Graimann, Michael Russold, Nejc Šarabon, Han Houdijk
}

\begin{abstract}
Most assistive robotic devices are exoskeletons which assist or augment the motion of the limbs and neglect the role of the spinal column in transferring load from the upper body and arms to the legs. In the SPEXOR project we will fill this gap and design a novel spinal exoskeleton to prevent low-back pain in able bodied workers and to support workers with low-back pain in vocational rehabilitation.
\end{abstract}

\section{CONTEXT}

Low-back pain (LBP) is a growing issue in our modern society. From all the diseases studied in the Global Burden of Disease Study from 2010 (Institute for Health Metrics and Evaluation), LBP was ranked number one concerning the years lived with disability in Europe [1]. Regarding the overall burden, LBP was ranked third in Central and Eastern Europe and first in Western Europe [1]. Depending on sex and region, the European point prevalence of LBP was up to $15.6 \%$. Prevalence and burden were found to increase with age and are even expected to further increase within the next years due to the current demographic developments [2]. From several studies reviewing the costs related to LBP in European countries, it has been calculated that the lowest cost per capita in Europe can be found in Belgium with $€ 116$ and $€ 209$ in Sweden [3]. Most of the costs of LBP are related to reduced work capacity, sick-leaves, early retirement and

J. Babič jan.babic@ijs.si is with the Department of Automation, Biocybernetics and Robotics, Jožef Stefan Institute, Slovenia; D. Lefeber is with the Department of Mechanical Engineering, Robotics \& Multibody Mechanics research group, Vrije Universiteit Brussel, Belgium; B. Graimann is with the Department of Translations Research and Knowledge Management, Otto Bock Healthcare GmbH, Germany; J. van Dieën is with the MOVE Research Institute Amsterdam, The Netherlands; H. Houdijk is with the Human Movement Sciences Amsterdam and with Heliomare Research and Development, The Netherlands; K. Mombaur is with the Department of Optimization in Robotics \& Biomechanics, Heidelberg University, Germany; M. Russold is with the Department of Translational Research, Otto Bock Healthcare Products GmbH, Austria; N. Sarabon is with the Department of Health Study, University of Primorska and with S2P Science To Practice d.o.o , Slovenia.

Members of SPEXOR consortium: J. Babič (coordinator), T. Petrič, R. Goljat: Jožef Stefan Institute, Slovenia; K. Mombaur (PI), M. Sreenivasa, M. Millard, P. Manns: Heidelberg University, Germany; D. Lefeber (PI), C. Rodriguez-Guerrero, L. De Rijcke, M. Näf: Vrije Universiteit Brussel, Belgium; J. van Dieën (PI), I. Kingma, G. Faber, S. Bruin, A. Koopman: VU University Amsterdam, The Netherlands; B. Graimann (PI), M. Tüttemann, A. Kurzweg, J. Bornmann, H. Glindemann: Otto Bock Healthcare GmbH, Germany; M. Russold (PI), D. Pieringer: Otto Bock Healthcare Products $\mathrm{GmbH}$, Austria; N. Šarabon (PI), A. Panjan, K. Kastelic, M. Savić: S2P Science to practice d.o.o., Slovenia; H. Houdijk (PI), C. van Bennekom (PI), J. Nachtegaal, S. Baltrusch: Heliomare, The Netherlands.

Funding: This project has received funding from the European Union's Horizon 2020 research and innovation programme under grant agreement No 687662 - SPEXOR.

Project webpage: http://www.spexor.eu

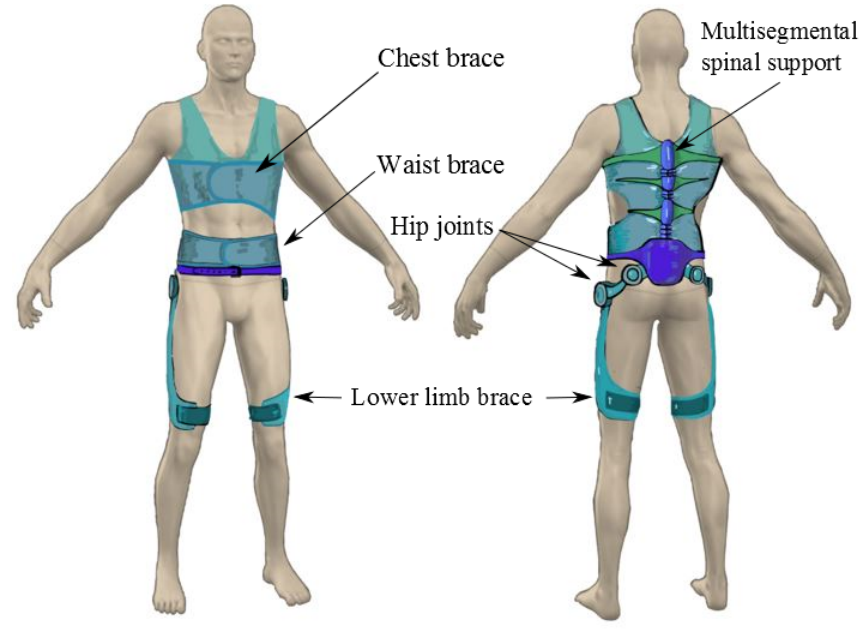

Fig. 1. A conceptual image of the SPEXOR exoskeleton [5]

legal claims. To reduce those costs and the burden on the workers, work place interventions such as education, exercise and braces, are becoming widely accepted. However, most of these measures ease the consequences of LBP but don't eliminate their causes. It has been shown that a strong correlation between physically demanding jobs and prevalence of LBP exists [4]. Movements which cause LBP in these jobs are: handling heavy loads, repeated lifting and turning, and working time spent in a flexed position. The SPEXOR project targets to design a spinal exoskeleton which assists these movements to prevent LBP for able-bodied workers and to support workers with LBP who are in vocational reintegration. Most of the existing assistive robotic devices are exoskeletons which augment the motion of the human's limbs. However, they mostly neglect the role of the spine in transferring the load from the upper body and limbs to the lower limbs, which will be the main contribution of the SPEXOR project.

\section{OBJECTIVES}

In the SPEXOR project, see figure 1, a spinal exoskeleton for working in sustained awkward postures and those involved in manual handling of heavy loads, will be designed. The exoskeleton will have several modes:

The assistive mode will prevent excessive or sustained non-neutral spine postures. It will also limit the mechanical load on the spinal column. 
The monitoring mode will monitor spinal movements and spinal loading and provide feedback to the user.

In the training mode, the device will provide active neuromuscular trunk conditioning. In this mode the exoskeleton will act as an advanced fitness or rehabilitation device to strengthen the wearer's muscles and prevent spine disorders.

Finally in the diagnostic mode the exoskeleton will provide an objective assessment of neuromuscular trunk functions. This mode will serve as a diagnostic tool to forecast possible physiological issues concerning the spine of the wearer.

The contribution of the authors of this paper will mainly be situated in the design and development of a flexible exoskeleton mechanism for spinal support. This mechanism has to be efficient as well as unobtrusive for the wearer. In this first design the emphasis will be on the requirements for LBP prevention and the use of passive viscoelastic elements for gravity compensation. The design will be made in a way to passively transfer external loads acting on the upper body and arms to the wearer's legs. In the second phase of the project the authors are responsible for the design and development of compliant actuators for this exoskeleton. The actuation will be designed to exhibit an adaptive compliant behavior. This will allow a comfortable interaction with the user and provide different levels of mechanical effort, depending on the need of torque augmentation, rehabilitation or training activities.

\section{GOING BEYOND THE STATE OF THE ART}

Several power augmenting and/or assistive exoskeletons have already been developed. From a technical point of view they can be split in two categories: active and passive devices. Although active devices are more powerful, they are also heavier as they require actuators and a power source. Examples of such active exoskeletons are the power assist suit for day-care from the Kanagawa Institute of Technology, JP [6], the lightweight active power assist device HAL from the Tsukuba University, JP [7], exoskeletons for the augmentation of soldier's combat abilities of the Defense Advanced Research Project Agency, USA, and the Lower extremity exoskeleton BLEEX, CA [8]. Recently an FP7 project named RoboMate has started. The RoboMate project is also targeting the issue of workspace injuries but, in contrast with the SPEXOR project, a full body exoskeleton is developed.

Passive devices are mainly used to reduce the burden of the user rather than to amplify the wearer's forces [9]. Some examples of such devices are the Smart Suite lite, a compact lightweight robotic suit which utilizes the elastomeric force of elastic materials to assist the wearer [9] and a passive device for enhancing limb movement for people with neuromuscular disabilities [10].

A crucial element in the development of active exoskeletons are the actuators. For the safety of the wearer a compliant behavior is desired [11]. Compliant actuators possess a physical elastic element capable of storing and releasing energy. Those actuators have an equilibrium position, which is the position where they do not exert any force/torque.
Deviations from this position are allowed. This allows a safer human-robot interaction, shock absorption, and can lead to a greater energy efficiency than with stiff actuators [12].

Although a lot of effort has already been spent on developing exoskeletons, only a few targeted the spine or lower back. The few which did, focused mostly on the assistance of the trunk flexion/extension [6].

To our knowledge, there is no exoskeleton which has been built yet that would provide assistance for functional trunk movements; an exoskeleton that doesn't only take power assistance into account but also the spine motions that could result in neuromuscular injuries and subsequently in LBP. This will be done in the SPEXOR project. The exoskeleton will be effective as assitive exoskeleton for laborers which have to work in awkward postures and repeatedly lift loads, as training device and as diagnostic tool. A wide range of possibilities for exercise and measurement protocols will be provided by SPEXOR. The combination of the different modes of the SPEXOR exoskeleton will provide a powerful preventive tool against the most frequent musculoskeletal disorder of today, LBP. This will make the spinal exoskeleton developed in SPEXOR a top-end device that goes well beyond the state-of-the-art.

\section{REFERENCES}

[1] R. Buchbinder, F. M. Blyth, L. M. March, P. Brooks, D. A. Woolf, and D. G. Hoy, "Placing the global burden of low back pain in context." Best Practice and Research: Clinical Rheumatology, vol. 27, pp. 575589, 2013.

[2] D. Hoy, L. March, P. Brooks, F. Blyth, A. Woolf, C. Bain, G. Williams, E. Smith, T. Vos, and J. Barendregt, "The global burden of low back pain: estimates from the Global Burden of Disease 2010 study," Annals of the rheumatic diseases, 2014.

[3] L. C. Lambeek, M. W. van Tulder, I. C. S. Swinkels, L. L. J. Koppes, J. R. Anema, and W. van Mechelen, "The trend in total cost of back pain in The Netherlands in the period 2002 to 2007." Spine, vol. 36, no. 13, pp. 1050-1058, 2011.

[4] G. Waddell and A. K. Burton, "Occupational health guidelines for the management of low back pain at work: Evidence review." Occupational Medicine, vol. 51, pp. 124-135, 2001.

[5] "Spinal Exoskeletal Robot for Low Back Pain Prevention and Vocational Reintegration." [Online]. Available: www.spexor.eu

[6] K. Yamamoto, M. Ishii, H. Noborisaka, and K. Hyodo, "Stand alone wearable power assisting suit-sensing and control systems," in International Workshop on Robot and Human Interactive Communication, 2004, pp. 661-666.

[7] H. Kawamoto and Y. Sankai, "Power assist method based on Phase Sequence and muscle force condition for HAL," Advanced Robotics, vol. 19, pp. 717-734, 2005.

[8] H. Kazerooni, "Hybrid Control of the Berkeley Lower Extremity Exoskeleton (BLEEX)," The International Journal of Robotics Research, vol. 25, no. 5-6, pp. 561-573, 2006.

[9] Y. Imamura, T. Tanaka, Y. Suzuki, K. Takizawa, and M. Yamanaka, "Motion-based design of elastic belts for passive assistive device using musculoskeletal model," in International Conference on Robotics and Biomimetics, 2011, pp. 1343-1348.

[10] T. Rahman, W. Sample, S. Jayakumar, M. M. King, J. Y. Wee, R. Seliktar, M. Alexander, M. Scavina, and A. Clark, "Passive exoskeletons for assisting limb movement," The Journal of Rehabilitation Research and Development, vol. 43, no. 5, p. 583, 2006.

[11] H. Vallery, J. Veneman, E. van Asseldonk, R. Ekkelenkamp, M. Buss, and H. van Der Kooij, "Compliant actuation of rehabilitation robots," IEEE Robotics and Automation Magazine, vol. 15, no. 3, pp. 60-69, 2008.

[12] B. Vanderborght, A. Albu-Schaeffer, A. Bicchi, E. Burdet, D. Caldwell, R. Carloni, and S. Wolf, "Variable impedance actuators: Moving the robots of tomorrow." in International Conference on Intelligent Robots and Systems, 2012, pp. 5454-5455. 